\title{
recildunds
}

Revista Científica Mundo de la Investigación y el Conocimiento

\author{
Juan Carlos Chancusig Chisag a; Milton Fabián Herrera Herrera ${ }^{\text {b; }}$ Mario Agustín \\ Banda Casa ${ }^{c}$; Hugo Ramiro Razo Ocaña ${ }^{d}$
}

Metodología e instrumentos aplicables a estudiantes de educación superior

Methodology and instruments applicable to higher education students

Revista Científica Mundo de la Investigación y el Conocimiento. Vol. 3 núm.3. Esp., noviembre, ISSN: 2588-073X, 2019, pp. 793-816

DOI: $10.26820 /$ recimundo/3.(3.Esp).noviembre.2019.793-816

URL: http://recimundo.com/index.php/es/article/view/630

Código UNESCO: 1105 Metodología

Tipo de Investigación: Artículo de Revisión

(C) RECIMUNDO; Editorial Saberes del Conocimiento, 2019

Recibido: $15 / 09 / 2019$

Aceptado: 23/10/2019

Publicado: 30/11/2019

Correspondencia: juan.chancusig@utc.edu.ec

a. Magister en Gestión de la Educación Mención en Educación Superior; Especialista en Diseño Curricular; Diploma Superior en Gestión Prospectiva de la Educación; Ingeniero en Informática y Sistemas Computacionales; Universidad Técnica de Cotopaxi; Cotopaxi, Ecuador; juan.chancusig@utc.edu.ec

b. Master en Ciencias de la Educación, mención en Planeamiento de Instituciones de Educación Superior; Diploma Superior en Didáctica de la Educación Superior; Licenciado en Ciencias de la Educación, Profesor de Enseñanza media en la Especialización de Biología y Química; Universidad Técnica de Cotopaxi; Cotopaxi, Ecuador; milton.herrera@utc.edu.ec

c. Magister en Gerencia Informática; Magister en Planeamiento y Administración Educativos; Diploma Superior en Didáctica de la Educación Superior; Ingeniero en Sistemas; Universidad Técnica de Cotopaxi; Cotopaxi, Ecuador; mario.banda@utc.edu.ec

d. Magister en Ciencias de la Educación mención Planeamiento y Administración Educativa; Especialista Superior en Gestión Local; Arquitecto; Universidad Técnica de Cotopaxi; Cotopaxi, Ecuador; hugo.razo@utc.edu.ec 


\section{Metodología e instrumentos aplicables a estudiantes de educación superior}

Vol. 3, núm. 3 Esp., (2019)

Juan Carlos Chancusig Chisag; Milton Fabián Herrera Herrera; Mario Agustín Banda Casa; Hugo Ramiro Razo Ocaña

\section{RESUMEN}

Los cambios presentes en la sociedad actual deben ser tomados en cuenta por los docentes universitarios para no quedarse pasivos; sino, todo lo contrario, participar de manera activa en la formación emprendedora del estudiante y convertirlo en un profesional útil, competente para el mundo laboral. Razón por la cual, la práctica andragógica en las aulas universitarias, debe ofrecer diferentes metodologías de enseñanza y aprendizaje e instrumentos de evaluación, a fin de fomentar competencias, que aporten pertinencia social a su desempeño profesional, es decir, ayudarlo a consolidar destrezas y habilidades que le ayuden a aceptar los nuevos retos de aprendizaje. Ante estas ideas, hay que incorporar una didáctica que aleje el predominio mecanicista e instrumental, para darle al hecho educativo una transformación constante, incluir las tecnologías, redes virtuales como pilares esenciales para elevar las capacidades interpretativas de los grupos y valorar el conocimiento desde una perspectiva cualitativa humana, que hace posible dejar a un lado aquellas evaluaciones medibles que establecen una ponderación sin llegar a estimar el verdadero saber alcanzado, es significativo el fomento de las competencias genéricas e intelectuales que consolidan el saber, habilidades y actitudes necesarias para realizar actividades diversas con un nivel de calidad y eficacia. Por lo tanto, es relevante la metodología a través del trabajo cooperativo, resolución de problemas, métodos complementarios de la acción didáctica al transformar los instrumentos tradicionales por nuevos esquemas centrados en el estudiante. La orientación investigativa del artículo corresponde a una revisión bibliográfica de tipo documental que tuvo como objetivo analizar la metodología e instrumentos aplicables a estudiantes de educación superior. En conclusión, para que el alumnado universitario muestre madurez académica se debe garantizar el aprendizaje, analizar no solamente las estrategias cognitivas, metacognitivas y de regulación que posee, sino la metodología docente, para propiciar cambios en los instrumentos aplicables a los estudiantes.

Palabras Claves: Metodología; Instrumentos; Educación superior. 


\title{
Metodología e instrumentos aplicables a estudiantes de educación superior
}

Vol. 3, núm. 3 Esp., (2019)

Juan Carlos Chancusig Chisag; Milton Fabián Herrera Herrera; Mario Agustín Banda Casa;

Hugo Ramiro Razo Ocaña

\begin{abstract}
University teachers so as not to remain passive; but quite the opposite to actively participate in the entrepreneurial training of the student and turn him into a useful professional, competent for the world of work, must take changes present in today's society into account. This is why andragogic practice in university classrooms should offer different teaching and learning methodologies and assessment tools, in order to promote skills, which bring social relevance to their professional performance, i.e., help you consolidate skills and skills to help you embrace new learning challenges. In the face of these ideas, we must incorporate a didactic that takes away the mechanistic and instrumental predominance, to give the educational fact a constant transformation, include technologies, virtual networks as essential pillars to elevate the capacities organizations and to value knowledge from a qualitative human perspective, which makes it possible to set aside those measurable assessments that establish a weighting without estimating the true knowledge achieved, the significant promoting generic and intellectual competences that consolidate the knowledge, skills and attitudes necessary to carry out diverse activities with a level of quality and effectiveness. Therefore, the methodology is relevant through cooperative work, problem solving, and complementary methods of didactic action in transforming traditional instruments by new student-centered schemes. The research orientation of the article corresponds to a documentary literature review that aimed to analyze the methodology and instruments applicable to higher education students. In conclusion, in order for university students to show academic maturity, learning must be ensured, not only the cognitive, metacognitive and regulatory strategies it possesses, but also the teaching methodology, to bring about changes in the instruments applicable to students.
\end{abstract}

Key Words: Methodology; Instruments; Higher education. 


\section{Metodología e instrumentos aplicables a estudiantes de educación superior}

Vol. 3, núm. 3 Esp., (2019)

Juan Carlos Chancusig Chisag; Milton Fabián Herrera Herrera; Mario Agustín Banda Casa; Hugo Ramiro Razo Ocaña

\section{Introducción.}

El proceso de acumulación de saberes se estimula desde los primeros signos de vida del ser humano. Está demostrado que el desarrollo cognitivo del individuo viene desde los primeros años de vida, las diferentes corrientes o enfoques pedagógicos generan teorías que permiten entender como los estudiantes al estar en una interacción con el docente y la presentación de saberes, logra entender e interpretar las diferentes informaciones que estructuran el currículo, de este modo, adquiere conocimientos útiles para su crecimiento personal, social y laboral que van a convertirse en medios importantes durante la adquisición de habilidades genéricas e intelectuales.

En consecuencia, le compete al docente universitario investigar las condiciones generales en las cuales descansa su enseñanza, para así ir de la mano hacia el progreso y desarrollo de cada nación. Hoy en día, el rol de la educación superior incluye el empleo de los conocimientos aprehendidos lo cual se traduce en el incremento del bienestar social. Además, para complementar su trabajo en el aula, tiene que observar el mundo globalizado, crecimiento de los países, desarrollo del conocimiento y aportes en materia de investigación científica originadas desde el sector educativo. Por ello, es de carácter relevante efectuar un análisis de las actividades y preparación en materia de metodología e instrumentos aplicables al estudiante a fin de contextualizar su trabajo bajo una visión integradora y competente que le ayude a fortalecer en los grupos nuevas actitudes y aptitudes en su aprendizaje.

Actualmente, la sociedad es tan cambiante y exigente en cuanto a aprendizajes, se hace necesario insistir que la educación superior efectúe su accionar de la mejor manera, para lograr 


\section{Metodología e instrumentos aplicables a estudiantes de educación superior}

Vol. 3, núm. 3 Esp., (2019)

Juan Carlos Chancusig Chisag; Milton Fabián Herrera Herrera; Mario Agustín Banda Casa;

Hugo Ramiro Razo Ocaña

su máximo desarrollo. En atención a lo señalado es oportuno la definición de interrogantes en cuanto a será necesario promover la innovación metodológica como indicador de generar los respectivos aportes que el docente debe ofrecer a sus estudiantes, mediante la aplicación de corrientes caracterizadas por reducir los efectos de la actividad mecanicista que existe en el proceso educativo universitario, entender que los alumnos poseen capacidades intelectuales para generar su propio conocimiento.

Por lo tanto, para propiciar nuevos procesos de enseñanza y aprendizaje en las universidades, el docente debe concebir su acción didáctica desde una perspectiva científica, con calidad, idónea a las habilidades de cada individuo, centrada en el estudiante, innovadora conducida mediante técnicas, métodos y recursos altamente calificados para lograr formar profesionales capaces de tomar decisiones acordes a las situaciones problemas encontradas en el entorno donde se circunscribe la educación. Al respecto, Pascuali (2016), señala en la actualidad "una buena parte del docente admite que la evaluación debe ser integral y no centrarse exclusivamente en la dimensión cognitiva del sujeto que aprende. (p.17). Es decir, este reconocimiento no significa que en su evaluación incluyan otro tipo de contenidos, los valores de aprendizajes, es verdad que existen algunas propuestas metodológicas interesantes al respecto, pero su empleo en la universidad debe estar generalizada, las prácticas de evaluación continúan siendo bastante rutinarias y estrechas.

De lo antes citado, se puede desglosar que mediante la implementación del proceso evaluativo, los docentes universitarios tienen oportunidad de introducir modificaciones al uso de los instrumentos requeridos para medir su aprendizaje, esto, permite girar de forma permanente cambios en la metodología, pues, se hace determinante identificar aquellas técnicas básicas en 


\section{Metodología e instrumentos aplicables a estudiantes de educación superior}

Vol. 3, núm. 3 Esp., (2019)

Juan Carlos Chancusig Chisag; Milton Fabián Herrera Herrera; Mario Agustín Banda Casa; Hugo Ramiro Razo Ocaña

correspondencia al instrumento por emplear, así como los respectivos métodos y recursos que darán a la práctica educativa la respectiva flexibilidad que amerita para responder a la visión y misión establecida.

De este modo, la evaluación debe ser vista por el docente universitario como un proceso constitutivo de cualquier propuesta didáctica; una oportunidad para promover una permanente reflexión sobre logros y obstáculos en el desarrollo de la tarea, un proceso sistemático, continuo e integrado dentro de un proceso más general que es el educativo. En otras palabras, representa un proceso que, a partir del conocimiento y comprensión de cierta información, permite emitir un juicio de valor acerca de un aspecto de la realidad en la cual se interviene en un determinado contexto socio histórico particular y a la vez posibilita tomar decisiones, desde el diálogo con quien esté involucrado, argumentar justificaciones del juicio de valor realizada.

En razón de las consideraciones anteriores, se puede decir que no es posible hoy referirse a la enseñanza universitaria al margen de los cambios actuales, los cuales, se convertirán en pautas a seguir por todas aquellas universidades que quieran estar entre las de mayor reconocimiento. Por ello, las orientaciones modernas hacen hincapié en la metodología como herramienta o palanca esencial para modificar los esquemas de los tradicionales contenidos académicos a contenidos profesionales centrados en el desarrollo de competencias y habilidades. Esto es, de una enseñanza basada en la información del profesorado a una enseñanza basada en la actividad formativa del estudiante. La creatividad, apoya las estrategias, técnicas, métodos y recursos innovadores orientados al aprendizaje, por cuanto es el alumno, el que ha de mostrar la adquisición de las competencias convenidas en cada una de las carreras. 


\section{Metodología e instrumentos aplicables a estudiantes de educación superior}

Vol. 3, núm. 3 Esp., (2019)

Juan Carlos Chancusig Chisag; Milton Fabián Herrera Herrera; Mario Agustín Banda Casa;

Hugo Ramiro Razo Ocaña

El sentido de globalización del aprendizaje es una consecuencia inmediata de esta transformación. Un profesional es una persona competente en su ámbito capaz de analizar y resolver los problemas y proponer mejoras (innovar). El profesor universitario es un profesional de la educación superior innovadora y creativa, con dominio del contenido formativo y estrategias didácticas, capaz de hacer que los alumnos se entusiasmen por aprender. Esta sería la clave para plantear la acción docente en la universidad. Tal como lo apoya, Violant (2018) las nuevas metodologías deben ser innovadoras y creativas.” (p.22). Dichas metodologías, buscan mediante estrategias entre otros aspectos, desarrollar capacidades y habilidades de ideación, interacción, elaboración, competencia comunicativa, argumentación para expresar y defender los propios puntos de vista, trabajo colaborativo, desempeño de roles. Se caracterizan por estar orientadas al desarrollo de actitudes, valores, sensibilidad emocional y persistencia en la tarea iniciada.

Bajo esta consideración, el profesor es algo más que un transmisor y evaluador de conocimientos. Hoy, resulta arcaica la imagen del profesor que lee la lección del libro de texto mientras los alumnos escuchan o escriben, del que se limita a dictar mientras los estudiantes copian, del que siempre como única estrategia la exposición. El profesor ha de organizar las tareas docentes con más variedad de metodologías centradas en estrategias y recursos didácticos, adaptadas los objetivos y necesidades del grupo clase para incorporar instrumentos ajustados a las características de la enseñanza y aprendizaje.

Cada uno de los planteamientos dados, permiten resaltar su importancia para el desarrollo del presente artículo, pues, se puede comprender que la actual función del docente universitario para lograr los objetivos planificados, debe concebir el desarrollo de sus actividades desde una 


\section{Metodología e instrumentos aplicables a estudiantes de educación superior}

Vol. 3, núm. 3 Esp., (2019)

Juan Carlos Chancusig Chisag; Milton Fabián Herrera Herrera; Mario Agustín Banda Casa; Hugo Ramiro Razo Ocaña

perspectiva metodológica enmarcada en estrategias, técnicas, métodos y recursos innovadores mediante los cuales, fomente la creatividad en los grupos, para así responder de forma específica a cada necesidad de aprendizaje visto en el aula, además, le asegura una selección de los instrumentos de manera viable a las características preestablecidas por la concepción metodológica.

\section{Metodología.}

Desde el punto de vista metodológico este artículo requiere la selección previa de una herramienta básica para darle continuidad a las consideraciones generales extraídas y asumidas como medios que dan cabida a otras interpretaciones subyacentes. En esta dirección, Sabino (2015) define al método "como una estrategia colectiva que facilita el desarrollo del trabajo, pues, amplía las acciones indagatorias hacia un final concluyente de aspectos metodológicos". (p.56). Por lo tanto, dar continuidad al proceso significa apoyarse en un adecuado método complementario que ayuda a revisar, interpretar y analizar los hechos en forma general.

En relación con este último planteamiento, se considera como método viable para el desarrollo del artículo, el tipo de concordancia conceptualizado por, García (2018) “como aquel que constituye un razonamiento acerca de las características, causas, basada en la comparación de las circunstancias que han acompañado varias veces la aparición del fenómeno" (p.66). Es decir, se tomaron en cuenta las diferentes definiciones o planteamientos expuestos por aquellos autores vinculados con el tema, para luego extrapolar sus interpretaciones hacia un contexto en particular y llegar finalmente a caracterizar el fenómeno en estudio.

\section{Tipo de Investigación}




\section{Metodología e instrumentos aplicables a estudiantes de educación superior}

Vol. 3, núm. 3 Esp., (2019)

Juan Carlos Chancusig Chisag; Milton Fabián Herrera Herrera; Mario Agustín Banda Casa;

Hugo Ramiro Razo Ocaña

Los aportes que se busca mediante el desarrollo del artículo, conduce a ubicarlo en una investigación documental, basada en la interpretación de diversas fuentes primarias, con la finalidad de contextualizar un problema particular. La misma responde a lo planteado por García (ob.cit), "como el estudio de problemas con el propósito de ampliar y profundizar el conocimiento de naturaleza de apoyo principalmente en trabajos previos, información, datos divulgados por medios impresos, audiovisuales o electrónicos.” (p.13).

Asimismo, Rangel (2018), “es un estudio de problemas con el propósito de ampliar y profundizar el conocimiento de su naturaleza con apoyo principalmente en trabajos previos, información, datos divulgados por medios impresos, audiovisuales o electrónicos.” (p.6). Esta definición, lleva a reforzar las apreciaciones anteriores, al considerar este tipo de investigación como una tarea exhaustiva que lleva al investigador a la elaboración de argumentos críticosreflexivos en cuanto a los diversos aspectos considerados en su desarrollado. De este modo, se entiende que, para lograr la interpretación de los hechos de manera precisa y coherente a las necesidades plasmados en su propósito, se requiere ubicarla en un nivel que según Arias (2014), "se refiere al grado de profundidad con que se aborda un objeto o fenómeno" (p.45). En consecuencia, para establecer la respectiva correspondencia con los hechos, se fija como nivel el descriptivo.

En consecuencia, las diferentes actividades cumplidas en el marco de la adecuación del método, tipo y nivel traen consigo importantes acciones que permiten establecer las distintas directrices capaces de orientar las posteriores tareas a cumplir antes y durante la revisión de los respectivos documentos que brindan particular información en materia del contenido temático seleccionado con antelación. 


\section{Metodología e instrumentos aplicables a estudiantes de educación superior}

Vol. 3, núm. 3 Esp., (2019)

Juan Carlos Chancusig Chisag; Milton Fabián Herrera Herrera; Mario Agustín Banda Casa; Hugo Ramiro Razo Ocaña

\section{Fuentes Documentales}

En cuanto a las actividades realizadas en esta fase, se puede considerar el planteamiento dado por, García (ob.cit), "los procedimientos son momentos activos que determinan las acciones que el investigador cumple durante el desarrollo del trabajo" (p.125). Es decir, se presentan las diferentes etapas o momentos que hicieron posible la realización de la investigación. Para lo cual, fue necesario recurrir a la implementación de una gama de técnicas documentales que dieron oportunidad a fijar posiciones claras en cuanto a la indagación para lograr una verdadera relación en los indicadores que estructuran el tema, requiere del apoyo de las fuentes teóricas que hacen posible entender y valorar la contextualización del fenómeno en el marco de una realidad visible de ser investigada.

\section{Técnica de Recolección de Información}

Con la finalidad de llegar a realizar interpretaciones críticas se fija como apoyo de la valoración crítica - reflexiva, la incorporación de un método viable y ajustado a las características que precisa cada contenido. Por ello, para cumplir con este momento, se hizo necesario la introducción de la técnica del resumen que consiste en seleccionar aspectos teóricos bajo un esquema interpretativo para luego extraer nuevas ideas, tal como la define, García (ob.cit), “consiste en la interpretación de contenidos en forma analítica"(p.66). Es importante acotar que mediante su incorporación se pudo no sólo seleccionar el material vinculante, sino, complementar aquellas ideas previas que permitieron dar la respectiva consistencia científica en el marco de una valoración flexible a los hechos o elementos que identifican el contenido a tratar. 


\section{Metodología e instrumentos aplicables a estudiantes de educación superior}

Vol. 3, núm. 3 Esp., (2019)

Juan Carlos Chancusig Chisag; Milton Fabián Herrera Herrera; Mario Agustín Banda Casa;

Hugo Ramiro Razo Ocaña

\section{Resultados.}

En función de la descripción de una situación con características viables para ser analizado, se puede indicar que dicha actividad permite fijar una postura crítica ante los diferentes aspectos encargados de conformar el contenido teórico del presente artículo, los mismos pretende darle al lector una visión general de aquellos argumentos esenciales para comprender la significación científica del tema previamente seleccionado.

\section{Metodología e Instrumentos Aplicables a los Estudiantes Universitarios}

Desde el escenario Latinoamericano la Educación Superior se ha destacado en el avance de sus actividades científicas; y especialmente en el realce de la investigación derivada en escritos novedosos; al efecto, una estrategia de innovación científica centrada en satisfacer los requerimientos y solicitudes de la sociedad para su progreso debe incluir a la universidad.

Se habla de innovación en el sentido de introducir algo nuevo y diferente; el término es empleado para designar un progreso con relación a la metodología estimada por métodos, materiales, formas de trabajo, entre otros utilizados con anterioridad, pero la mejora por si sola puede, o no, ser innovación. En consecuencia, existe una relación entre los términos innovación y cambio, por cuanto, si la innovación significa la introducción de algo nuevo que genera progresos, el hecho de pasar de lo que se tenía antes, a un estado de mejoría, supone la presencia de un cambio.

El desarrollo acelerado de la sociedad y las tendencias actuales del aprendizaje, obligan a la Educación Superior a cumplir con nuevos roles; el profesor deja de ser la fuente de 


\section{Metodología e instrumentos aplicables a estudiantes de educación superior}

Vol. 3, núm. 3 Esp., (2019)

Juan Carlos Chancusig Chisag; Milton Fabián Herrera Herrera; Mario Agustín Banda Casa; Hugo Ramiro Razo Ocaña

conocimiento y pasa a ser, un guía para sus estudiantes, facilitándoles el uso de los recursos y herramientas que necesiten para explorar y elaborar nuevos conocimientos y destrezas. Estos cambios en la Educación Superior guardan relación directa con la metodología, permitiéndole con una actitud crítica y reflexiva ante las solicitudes sociales, transformar la información en conocimiento, orientar el proceso de aprendizaje y lograr en el individuo una formación integral para vivir y convivir en la sociedad actual.

En razón de lo anterior, al hacer referencia a la metodología e instrumentos aplicables al estudiante representa una habilidad y actitud ante las personas y hechos, el profesor creativo posee unas características en las tres dimensiones presentes en educación: ser, saber y hacer. Dicho con otras palabras, actitudes flexibles, dominio de los contenidos y su adaptación a los destinatarios, habilidad didáctica. A continuación, se describe la actuación metodológica del docente:

El profesor innovador y creativo posee una disposición flexible hacia las personas, las decisiones y los acontecimientos; no sólo tolera los cambios sino que está abierto a ellos más que otras personas; está receptivo a ideas y sugerencias de los otros, ya sean superiores, compañeros o inferiores; valora el hecho diferencial; se adapta fácilmente a lo nuevo sin ofrecer excesivas resistencias; se implica en proyectos de innovación.

Por lo que respecta a su capacidad o conocimiento, la percepción rica en matices de cuanto le rodea. No se queda con la idea general, sino que relaciona fácilmente un hecho con otro y unas ideas con otras. En esta misma línea cabe destacar su facilidad para integrar y evocar 


\section{Metodología e instrumentos aplicables a estudiantes de educación superior}

Vol. 3, núm. 3 Esp., (2019)

Juan Carlos Chancusig Chisag; Milton Fabián Herrera Herrera; Mario Agustín Banda Casa;

Hugo Ramiro Razo Ocaña

experiencias. Conoce y aplica diversas técnicas orientadas a la ideación y la creatividad de sus alumnos, no contentándose con que estos repitan lo que han oído o estudiado.

Entre las habilidades podemos referirnos a actuaciones como: inducir a los sujetos para que se sensibilicen a los problemas; promover el aprendizaje por descubrimiento; crear un clima de seguridad y fácil comunicación entre las personas; incitar al sobre-aprendizaje y autodisciplina; diferir el juicio crítico cuando se están exponiendo ideas; estimular los procesos divergentes; formular e incitar a las preguntas divergentes; aplicar técnicas creativas. Estas actitudes son claves para generar climas de autoaprendizaje y de implicación espontánea y colaborativa. Su actuación en tanto que profesional innovador y creativo de la enseñanza, la resumiríamos en su habilidad para entusiasmar e inducir a los estudiantes hacia el autoaprendizaje, hacerles tan atractivo y sorprendente el contenido que sean capaces de emplear en aprender más tiempo del habitual sin que ello les incomode.

Al contrario, disfrutan aprendiendo porque hacen aportaciones personales, porque crean o recrean los aprendizajes, porque existe un reconocimiento externo y una satisfacción interna. Bajo estas consideraciones, la creatividad docente radica en dejar huella, dejar impronta, de modo que pasado el tiempo aún se recuerda a aquellos maestros o profesores que nos trasmitieron algo más que información. Nos dejaron ese mensaje humano, clima, espíritu, impacto, que con el tiempo quedó en nosotros como huella modélica permanente.

En esta dirección, Torres (2017), precisa que "los docentes universitarios para dar cambios a la actividad metodológica deben concebir el hecho educativo de manera global". (p.21). Según lo planteado, se puede entender que la acción educativa en el marco de la 


\section{Metodología e instrumentos aplicables a estudiantes de educación superior}

Vol. 3, núm. 3 Esp., (2019)

Juan Carlos Chancusig Chisag; Milton Fabián Herrera Herrera; Mario Agustín Banda Casa; Hugo Ramiro Razo Ocaña

educación superior, debe concebir el proceso desde una visión generadora de cambios específicos en los estudiantes.

Por lo tanto, es significativo considerar en la organización de la planificación educativa, la actuación de una metodología caracterizada por estrategias, al igual que métodos o procedimientos, y cualquier actuación formativa, encuentran su razón de ser en la meta perseguida. Incluso los grupos y organizaciones se cohesionan y mantienen en el tiempo gracias a compartir determinados fines que intentan conseguir. Alcanzados estos, el grupo pasa por momentos de crisis y descomposición. Las estrategias se concretan a la luz de las finalidades y objetivos. Por eso carece de sentido hablar de la bondad o pertinencia de las estrategias en general, al margen de lo que se pretende.

Otro componente de importancia en la metodología lo representa convertir a la estrategia en flexible y creativa es la secuencia adaptativa. Eso significa que el diseño inicial puede sufrir modificaciones en función de los sujetos, contenidos, condiciones espacio temporales, agrupamientos, situaciones nuevas que aparecen a lo largo del proceso. En diversas ocasiones las estrategias docentes utilizadas con un turno o grupo funcionan y con otro no. Una estrategia tiene tanto de sucesión planificada de acciones como de indeterminación sociológica. La realidad contextual es sin duda un elemento clave por cuanto sitúa la teoría y acción en la realidad concreta, en la pura complejidad de los hechos que confluyen decenas de variantes. La valoración del contexto tal vez sea el componente más sustantivo y esencial de la estrategia frente a otros conceptos mediadores como método, procedimiento, técnica en los que predomina la secuencia encadenada. El contexto es el referente de partida, de proceso y de llegada. 


\section{Metodología e instrumentos aplicables a estudiantes de educación superior}

Vol. 3, núm. 3 Esp., (2019)

Juan Carlos Chancusig Chisag; Milton Fabián Herrera Herrera; Mario Agustín Banda Casa;

Hugo Ramiro Razo Ocaña

Por lo tanto, al considerar la metodología de investigación basada en el desarrollo como el proceso de construcción del conocimiento en el que se retoman en diferentes momentos y de forma interactiva y recursiva los objetivos, las estrategias y valoraciones con el fin de aproximarse al estudio del cambio. Este procedimiento de carácter abierto y recursivo es válido tanto para la construcción individual como colectiva del conocimiento por cuanto uno y otro son fruto de la relación entre los conceptos ya adquiridos y los nuevos conceptos a través de los estímulos del medio.

Según Alvarado (2015) emplear la metodología basada en el desarrollo significa tomar el cambio como referente y por lo mismo será el principal foco de atención; el procedimiento seguido no es lineal sino recursivo, volviendo sobre sí cuantas veces sea necesario; nunca son definitivos los instrumentos y muestras; las etapas temporales son sustituidas por momentos o situaciones que hacen de hitos en la investigación; es un proceso dinámico y el grupo avanza modificando los resultados que va generando; caracterizado por la recursividad e interactividad permanente; que la investigación comporta por sí misma un proceso formativo.

En el campo de esta metodología, los instrumentos nunca son definitivos, muestras y recursos de recogida de información, sino que un nuevo objetivo puede comportar la recogida de nueva información modificando la muestra inicial. En tal sentido se encuentra con pluralidad de muestras, instrumentos y resultados a lo largo del proceso en función de los momentos. La misma se sucede en el tiempo a través de momentos. Más que estadios o fases de tiempo predeterminado y fijo, secuencia su actividad a través de momentos de duración irregular. El momento coincide con el periodo de revisión y actualización de lo que se va realizando. 


\section{Metodología e instrumentos aplicables a estudiantes de educación superior}

Vol. 3, núm. 3 Esp., (2019)

Juan Carlos Chancusig Chisag; Milton Fabián Herrera Herrera; Mario Agustín Banda Casa; Hugo Ramiro Razo Ocaña

\section{Imagen 1. Componentes de la Metodología en Desarrollo}

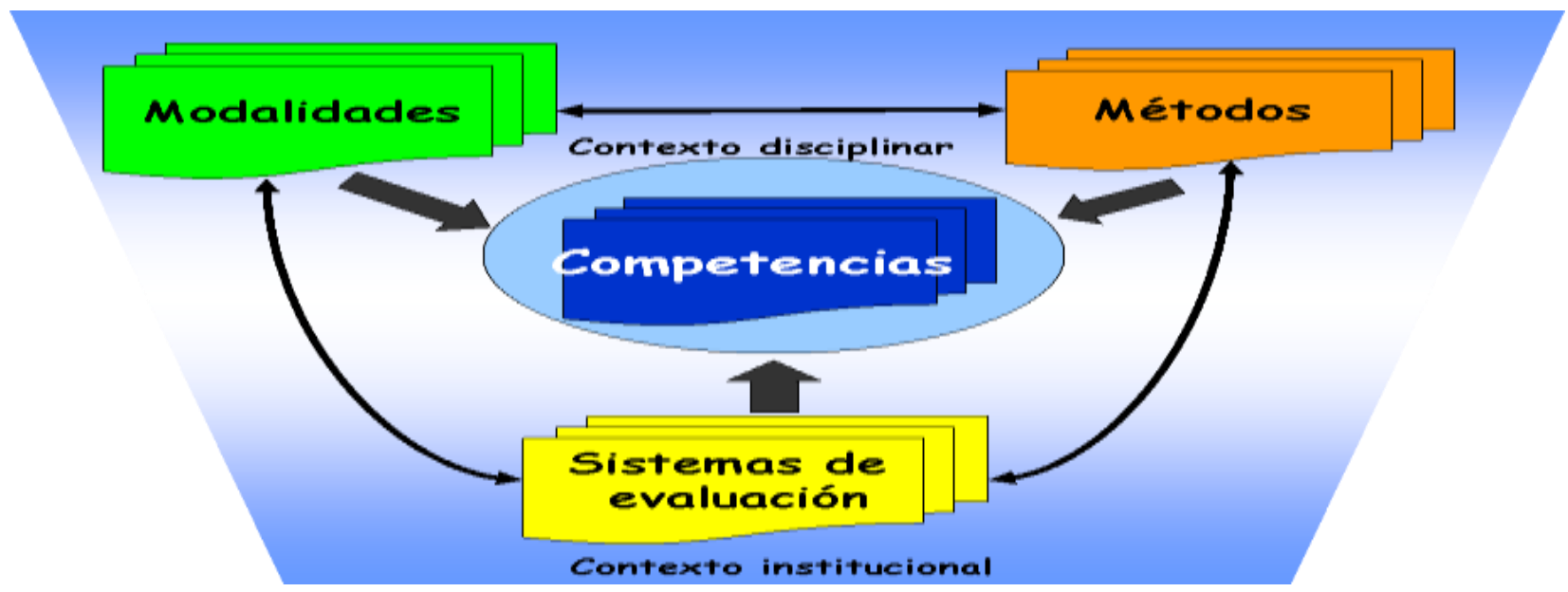

Fuente: Torres (ob.cit)

Al describir la imagen relativa a la metodología de desarrollo, se encuentra que los docentes universitarios, deben partir de las modalidades donde está enmarcada la acción educativa, es decir, presencial o virtual, asimismo, el contexto disciplina relacionado con el área de conocimiento dirigida a práctica, debe ser vista como un aspecto global del proceso de enseñar, apoyado en métodos que deben estar en correspondencia con las técnicas, estrategias, recursos y la respectiva adaptabilidad de los instrumentos que surgen en la medida que cada estudiante asume el aprendizaje desde sus propias competencias.

En consecuencia, toda discusión didáctica, la evaluación de aprendizajes resulta un eje central sobre el cual no siempre hay acuerdos ni miradas convergentes. Se parte de la evaluación considerada como parte integral del proceso de aprendizaje, como un proceso continuo y participativo que permite identificar una problemática, analizarla y explicarla mediante 


\section{Metodología e instrumentos aplicables a estudiantes de educación superior}

Vol. 3, núm. 3 Esp., (2019)

Juan Carlos Chancusig Chisag; Milton Fabián Herrera Herrera; Mario Agustín Banda Casa;

Hugo Ramiro Razo Ocaña

información relevante y es parte constitutiva de cualquier propuesta didáctica. Es una oportunidad para promover una permanente reflexión sobre logros y obstáculos en el desarrollo de la tarea. Implica un proceso sistemático, continuo e integrado dentro de un proceso más general que es el educativo.

Asimismo, se puede resaltar que la tecnología está cambiando el escenario educativo, las tecnologías de la información y la comunicación son herramientas para agilizar, optimizar y extender procesos de enseñanza y aprendizaje. La influencia de la nueva tecnología en los modos de aprender genera nuevos desafíos para la evaluación. En este sentido las plataformas de elearning ofrecen diversidad de herramientas y propician la adopción de distintos tipos de estrategias, tanto para el desarrollo de los contenidos como para las propuestas de actividades que favorecen la construcción del aprendizaje y su evaluación. Apoyado en las ideas expuestas por, Lezcano (2016), las incorporaciones de las TIC a los procesos de enseñanza y de aprendizaje en la era digital están modificando los ambientes tradicionales de enseñanza tanto presenciales como a distancia. La principal diferencia entre estas dos modalidades se centra en la separación física o no entre profesores y alumnos.

En razón de lo anterior, la integración de las posibilidades de la comunicación mediada por ordenador (correo electrónico, foros de discusión, chat, videoconferencia, mensajería instantánea tipo MSN, twitter, redes sociales, entre otros) diluye estas diferencias y permite un abanico de experiencias de aprendizaje que van desde aulas convencionales unidas a través de la red a grupos de trabajo colaborativo en contextos totalmente a distancia. Las nuevas modalidades educativas vienen definidas no tanto por la separación física entre profesores y alumnos entre sí, sino por cantidad y calidad de diálogo entre ellos y por la flexibilidad del diseño de los cursos en 


\section{Metodología e instrumentos aplicables a estudiantes de educación superior}

Vol. 3, núm. 3 Esp., (2019)

Juan Carlos Chancusig Chisag; Milton Fabián Herrera Herrera; Mario Agustín Banda Casa; Hugo Ramiro Razo Ocaña

cuanto a objetivos, estrategias de aprendizaje y métodos de evaluación. Esto supone la construcción de nuevos espacios de comunicación que posibilitan una gran variedad de situaciones comunicativas, situaciones que tantos profesores como estudiantes deben acomodar, apropiarse, dominar, para que se produzca el aprendizaje, la construcción personal del conocimiento, la realidad del conocimiento compartido.

Los cambios en las prácticas, en la forma de desenvolverse de profesores y alumnos en estos nuevos espacios comunicativos pueden ser considerados como verdaderos cambios de su rol en los procesos de enseñanza y de aprendizaje. Aquí se abordan dichas prácticas en un escenario muy concreto, los entornos virtuales de enseñanza-aprendizaje, conscientes de que no es el único ni en algunos casos, el más adecuado para desarrollar secuencias de aprendizaje.

Las plataformas de e-learning ofrecen diversidad de herramientas y propician la adopción de distintos tipos de estrategias docentes, tanto para el desarrollo de los contenidos como para las propuestas de actividades que favorecen la construcción del aprendizaje y su evaluación. Para identificar el avance de los procesos cognitivos e interactivos, es necesario contar con una estrategia flexible de evaluación, así como una serie de herramientas que permitan detectar cambios en la complejidad de las construcciones de conocimientos por parte de los estudiantes. El rol que la evaluación tiene en la formación on line obliga a una planificación y ejecución minuciosa y el análisis atento de cada uno de sus elementos.

En ambientes mediados por tecnología, según Lezcano (ob.cit), es fundamental que la evaluación sea continua en base a diferentes instrumentos que permitan apreciar el avance da cada estudiante en los distintos niveles y ejes temáticos por los que transita al construir su 


\section{Metodología e instrumentos aplicables a estudiantes de educación superior}

Vol. 3, núm. 3 Esp., (2019)

Juan Carlos Chancusig Chisag; Milton Fabián Herrera Herrera; Mario Agustín Banda Casa;

Hugo Ramiro Razo Ocaña

conocimiento. Al diseñar la evaluación on-line deben considerarse las características del aprendizaje derivadas de sus fundamentos: constructivista, basado en recursos, colaborativo, basado en problemas, situado, entre otras. Se requiere una evaluación congruente con la concepción del proceso de aprendizaje de partida. De ahí la importancia de interesarse por los fundamentos pedagógicos y de incluir la evaluación en los cambios metodológicos asociados al uso de estrategias centradas en el alumno mediante TIC. De nada sirven éstas, si no se producen cambios también en la evaluación.

Este mismo autor, establece una distinción entre evaluación tradicional y evaluación alternativa. En el primer caso prevalece la evaluación separada de la enseñanza y aprendizaje, no se establecen criterios para evaluar, escasa variedad de instrumentos de evaluación, poder y control de la evaluación residen en el docente y se reconoce la fiabilidad y validez de los instrumentos usados para evaluar. En cuanto a la evaluación alternativa, los procesos de enseñanza y evaluación están integrados como un todo, criterios de evaluación acordados y explicitados, variedad de métodos de fuentes de información, poder compartido entre docentes y estudiantes, fiabilidad y validez es un elemento más en el proceso. Esta postura intenta superar la postura que considera la evaluación como un simple proceso que propone la realización de pruebas y exámenes.

En el marco de las metodologías dialógicas el rol que la evaluación tiene en la formación en ambientes obliga a una planeación y ejecución minuciosa y el análisis atento de cada uno de sus elementos: cada evaluación conlleva una retroalimentación casi inmediata, esto implica un paso hacia una educación centrada en el aprendizaje, con énfasis en la construcción del aprendizaje. Desde este marco, la evaluación de aprendizajes no debe ser una mera reutilización 


\section{Metodología e instrumentos aplicables a estudiantes de educación superior}

Vol. 3, núm. 3 Esp., (2019)

Juan Carlos Chancusig Chisag; Milton Fabián Herrera Herrera; Mario Agustín Banda Casa; Hugo Ramiro Razo Ocaña

de instrumentos de evaluación de la modalidad presencial sino la búsqueda de formas nuevas de evaluar, acordes tanto al entorno virtual como las competencias y capacidades a evaluar. Los instrumentos conocidos pueden servir como punto de partida, pero se debe asumir el desafío que el cambio sea de raíz y que el encuadre de la propuesta alcance a todos los componentes de la planificación didáctica. Para identificar el proceso de avance de los procesos cognitivos e interactivos de aprendizaje es necesario contar con una estrategia sensible y válida de evaluación de desempeños, así como una serie de herramientas que permitan detectar cambios en la complejidad de las construcciones por parte de los estudiantes.

Un instrumento de importancia en el campo de la metodología educativa universitaria lo representa e portafolio, conocido como cuaderno de trabajo, reflejan el proceso de aprendizaje a través de la recopilación de evidencias de ese trayecto. Como instrumento de evaluación posee diversas aplicaciones. Colabora en la medición de aspectos del aprendizaje que no son medibles a través de pruebas escritas, favorece en el estudiante la toma de conciencia de sus logros, de los aprendizajes alcanzados, como así también de los obstáculos que se presentaron en el proceso. Además, ayuda a que el estudiante sea protagonista de su aprendizaje y monitoree sus progresos y dificultades.

En relación a los foros, se puede decir que permiten trabajar en forma asincrónica con los estudiantes, visualizar y acompañar la construcción del conocimiento. El andamiaje pedagógico está dado por la devolución a un planteo, el pedido de revisión, una pregunta y/o repregunta que obliga a revisar posicionamientos, reflexionar, deliberar y tomar postura. Para los docentes implica un trabajo de seguimiento y monitoreo de las intervenciones de los estudiantes para orientar o reorientar si fuera necesario este proceso. Para que puedan asumirse como 


\section{Metodología e instrumentos aplicables a estudiantes de educación superior}

Vol. 3, núm. 3 Esp., (2019)

Juan Carlos Chancusig Chisag; Milton Fabián Herrera Herrera; Mario Agustín Banda Casa;

Hugo Ramiro Razo Ocaña

instrumentos de evaluación que facilitan la interactividad deben propiciar la resolución de problemas, la participación de todos los estudiantes, el compartir ideas, analizar opiniones y reflexiones. Estos son componentes muy valiosos al evaluar los propios aprendizajes y los ajenos, se rescatan las intervenciones de los docentes o tutores como andamiajes, como trampolines que facilitan la construcción del aprendizaje.

\section{Educación Superior}

Actualmente existe preocupación por parte de los actores inmersos en la universidad, acerca de la relevancia de la Educación Superior en países de América Latina y de los aportes de la misma a su contexto inmediato, a favor de solventar algunas exigencias de la colectividad. De allí que puede inferirse que hoy día, se aprecia una dinámica de transformación desde la Educación Superior en las naciones de Latinoamérica; desde los espacios donde el individuo se desenvuelve.

Al considerar lo anterior, Malagon (2015) indica que "en distintos países del mundo se plantea la urgente necesidad de transformar la educación en atención a las exigencias del tiempo que se vive actualmente". (p. 18). Países diversos en América Latina consideran que las universidades en general deben estar alertas a los acontecimientos fuera de las aulas donde se despliegan sus acciones, es decir, alertas a las actividades a resolver en el espacio social.

Dada la sociedad del conocimiento, donde nos encontramos, es necesario que la universidad ejecute cambios donde ofrezca equidad a los individuos de la sociedad; por ello, la educación superior es el punto fuerte donde se genera y emite conocimiento a las personas para un mejor futuro. En función a esto, es en la universidad donde se requiere afianzar los procesos 


\section{Metodología e instrumentos aplicables a estudiantes de educación superior}

Vol. 3, núm. 3 Esp., (2019)

Juan Carlos Chancusig Chisag; Milton Fabián Herrera Herrera; Mario Agustín Banda Casa; Hugo Ramiro Razo Ocaña

de instrucción como reto indelegable en cuanto a conocimiento impartido; destacándose la relevancia de modificar su praxis académica hacia una metodología ajustada a la realidad social.

La universidad Latinoamericana en general, centra su finalidad en preparar y formar personas idóneas competentes para solucionar requerimientos existentes en una sociedad inmersa en permanente transformación; y donde estos profesionales deben tomar decisiones adecuadas al momento. En virtud de las diversas exigencias sociales, le corresponde a la Educación Superior innovar en su quehacer pedagógico y administrativo, por lo cual es corresponsable de generar conocimientos para ello. En definitiva, la Educación Superior le compete formar parte de la realidad social y ello tomando en cuenta aspectos investigativos como aspecto vivencial y difusor de conocimiento.

\section{Conclusiones.}

Los cambios registrados a nivel mundial, son los encargados de proyectar nuevos aportes en materia educativa, como lo representa el contenido de tema antes abordado, el mismo hizo posible recorrer diferentes apreciaciones para plantear a continuación las consideraciones siguientes:

Como corresponsable en el progreso social de la colectividad, la universidad debe asumir su disposición en la búsqueda de propuestas que satisfagan los requerimientos colectivos, de tal manera que los desafíos o retos a los cuales se enfrenta la sociedad pueda tomarlos satisfactoriamente. Los cambios en los paradigmas educativos, propiciados por los avances científicos y tecnológicos en diversos campos, apuntan hacia una restructuración del significado del personal docente y su necesaria constitución como facilitador de procesos de aprendizaje. 


\section{Metodología e instrumentos aplicables a estudiantes de educación superior}

Vol. 3, núm. 3 Esp., (2019)

Juan Carlos Chancusig Chisag; Milton Fabián Herrera Herrera; Mario Agustín Banda Casa;

Hugo Ramiro Razo Ocaña

De esta manera, la concepción del profesor como transmisor y del estudiante como receptor de conocimientos es sustituida por la concepción del docente como orientador, guía que acompaña al estudiante en el proceso de construcción no sólo de conocimientos, sino también en el desarrollo de habilidades y valores asociados a un desempeño profesional eficiente, ético y responsable, del estudiante como sujeto de aprendizaje. Por ello, se hace determinante un cambio en la metodología y por consiguiente en los instrumentos aplicables a los estudiantes.

Asimismo, se precisa que cuando el docente incluye en su planificación los entornos virtuales como estrategias, su método didáctico debe buscar la retroalimentación como indicador de que cada estudiante ha logrado el aprendizaje de manera individual pero mediante procesos colaborativos e interactivos que le ayudan a consolidar sus capacidades cognitivas, intelectuales y sociales.

Por ello, los instrumentos de evaluación que se emplean permiten un mayor acercamiento al objeto de aprendizaje evaluado, brindando más y mejor información sobre las aristas del proceso evaluado. El análisis sobre los instrumentos como parte de una propuesta didáctica integral genera la posibilidad y necesidad de repensar que es y cómo deben llevarse los procesos de retroalimentación como parte de la estrategia evaluativa en los entornos virtuales institucionales. La evaluación es más que la consideración de los instrumentos y las evidencias relevadas. La comunicación, interacción y retroalimentación son las protagonistas; los instrumentos pueden ser facilitadores de estos procesos.

Estos procesos de cambio hacia una evaluación que promueva el uso de instrumentos que favorezcan la retroalimentación como parte del aprendizaje y desarrollo integral de los 


\section{Metodología e instrumentos aplicables a estudiantes de educación superior}

Vol. 3, núm. 3 Esp., (2019)

Juan Carlos Chancusig Chisag; Milton Fabián Herrera Herrera; Mario Agustín Banda Casa; Hugo Ramiro Razo Ocaña

estudiantes requiere una transformación importante de muchos factores retroalimentación debe requerir una acción del estudiante, con miras a los criterios de evaluación, que lo involucre en su aprendizaje y lo comprometa a avanzar.

\section{Bibliografía.}

Alvarado, S. (2017). Técnicas de Enseñanza. Barcelona: Octaedro.

Arias, F. (2014). El Proyecto de Invvestigación. Caracas: Episteme .

García, P. (2016). Investigación. Caracas: Sypal.

I, L. (2016). Estrategias Virtuales. España: Narcea.

Malagón, C. (2015). Paradigmas de las Universidades. Sevilla: Didáctica.

Pascuali, S. (2016). Desarrollo Profesional. México: Balbao.

Sabino, C. (2015). El Proceso de Investigación. Caracas: Panapo.

Torres, M. (2017). Diversidad Creativa. Barcelona: Paidós.

Violant, P. (2018). Instrumentos de Ealuación. Madrid: Morata.

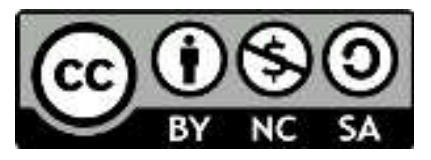

RECONOCIMIENTO-NOCOMERCIAL-COMPARTIRIGUAL

CC BY-NC-SA

ESTA LICENCIA PERMITE A OTROS ENTREMEZCLAR, AJUSTAR Y CONSTRUIR A PARTIR DE SU OBRA CON FINES NO COMERCIALES, SIEMPRE Y CUANDO LE RECONOZCAN LA AUTORÍA Y SUS NUEVAS CREACIONES ESTÉN BAJO UNA LICENCIA CON LOS MISMOS TÉRMINOS. 